\title{
RR Lyrae: Shock waves and atmospheric motions.
}

\author{
Agnès Lebre \\ DASGAL, Observatoire de Meudon, 92195 Meudon Cedex, France
}

\begin{abstract}
New high resolution spectroscopic observations of the star RR Lyrae are reported (resolution between 15,000 and 20,000). The spectra were taken in July 1990 with the $1.52 \mathrm{~m}$ telescope of the Observatoire de Haute Provence, using the AURELIE spectrograph, and were centered around $\mathrm{H} \beta$ and $\mathrm{Na} D$. They were distributed in time through almost an entire composite cycle $(P=13.6$ hours). Photospheric Fe $I$ lines were analysed and their FWHM variations over one luminosity cycle are interpreted in the framework of the propagation of shock waves running through the low atmospheric layers.
\end{abstract}

From previous works (Gillet \& Crowe, 1988 and 1989; Hill 1972; Fokin, 1992) striking features (the Hump and the Bump) on RR Lyrae light curves are (respectively) associated with two shock waves, generated during each pulsational cycle: the main strong shock, propagating outward (consequence of the $\kappa$-mechanism), and the early weak shock, resulting from the fast infall motion due to the large ballistic motion produced by the previous main shock.

From our new spectra we present for $\mathrm{Fe} I \lambda \lambda 4918.999 \AA$ and $4920.509 \AA$ photospheric lines the heliocentric radial velocity and the FWHM variations curves. On these latter, we clearly detect two peaks corresponding to important enlargments of the lines occuring at two phases in the pulsational cycle: a main and narrow peak occuring at the hump phase which is a first detection at the photospheric level of the outward propagating main shock; and a second wider peak occuring at the bump phase which already indicates the presence of a compression wave at the photospheric level. This result provides an important observational constraint to the theoretical developments devoted to RR Lyrae atmospheres that must take into account these enlargements observed on $\mathrm{Fe} I$ lines confirming the presence at the photospheric level of the main and early shocks.

\section{References:}

Fokin A.B., 1992, M.N.R.A.S., submitted.

Gillet D. \& Crowe R.A., 1988, Astron.\& Astrophys., 199, 242.

Gillet D. \& Crowe R.A., 1989, Astron.\& Astrophys., 225, 445.

Hill S.J., 1972, Astrophys.J., 178, 793. 